\title{
Localizing B-lines in Lung Ultrasonography by Weakly-Supervised Deep Learning, in-vivo results
}

\author{
Ruud JG van Sloun, Member, IEEE and Libertario Demi, Member, IEEE
}

\begin{abstract}
Lung ultrasound (LUS) is nowadays gaining growing attention from both the clinical and technical world. Of particular interest are several imaging-artifacts, e.g., A- and B- line artifacts. While A-lines are a visual pattern which essentially represent a healthy lung surface, B-line artifacts correlate with a wide range of pathological conditions affecting the lung parenchyma. In fact, the appearance of B-lines correlates to an increase in extravascular lung water, interstitial lung diseases, cardiogenic and non-cardiogenic lung edema, interstitial pneumonia and lung contusion. Detection and localization of Blines in a LUS video are therefore tasks of great clinical interest, with accurate, objective and timely evaluation being critical. This is particularly true in environments such as the emergency units, where timely decision may be crucial. In this work, we present and describe a method aimed at supporting clinicians by automatically detecting and localizing B-lines in an ultrasound scan. To this end, we employ modern deep learning strategies and train a fully convolutional neural network to perform this task on B-mode images of dedicated ultrasound phantoms in-vitro, and on patients in-vivo. An accuracy, sensitivity, specificity, negative and positive predictive value equal to $0.917,0.915,0.918,0.950$ and 0.864 were achieved in-vitro, respectively. Using a clinical system in-vivo, these statistics were $0.892,0.871,0.930,0.798$ and 0.958 , respectively. We moreover calculate neural attention maps that visualize which components in the image triggered the network, thereby offering simultaneous weakly-supervised localization. These promising results confirm the capability of the proposed method to identify and localize the presence of B-lines in clinical lung ultrasonography.
\end{abstract}

Index Terms-Lung Ultrasound Imaging, B-lines, Deep Learning, Class Activation Mapping

\section{INTRODUCTION}

Despite the strong artefactual nature of lung ultrasound images, which is due to the combined presence of air and soft tissue in the field of view and by the lack of signal processing techniques designed around the peculiarities of lung tissue, the application of ultrasound imaging to the diagnosis and monitoring of lung diseases is nowadays receiving growing attention from both the clinical and technical world. Specifically, well defined imaging artifacts are of great clinical interest, e.g., A- and B-line artifacts [1], [2], [3], [4], [5], [6], [7]. A-lines can be described as multiple equidistant hyper-echoic horizontal lines, which are generally visualized

Ruud JG van Sloun is with the Department of Electrical Engineering, Eindhoven University of Technology, 5612 AZ, Eindhoven, the Netherlands (email: r.j.g.v.sloun@tue.nl)

Libertario Demi is with the Department of Information Engineering and Computer Science, University of Trento, Via Sommarive 9, 38123, Trento, Italy (email:libertario.demi@unitn.it) across the entire image and that are parallel to the pleuraline. Essentially, they represent the normal pattern of the lung if pneumothorax is excluded. The genesis of A-lines is explainable by the fact that a healthy lung, formed by a cloud of closely packed 200-300 microns large air-bubbles (i.e. the alveoli), behaves essentially as a perfect reflector to ultrasound waves [4], [5]. Consequently, the ultrasound pulse emitted by the probe can be reflected multiple times between the probe and the soft-tissue/air interface, i.e. the pleura-line, before its amplitude is such that is no longer sufficient to be visualized in the ultrasound image. Differently, B-line artifacts, defined as hyper-echoic vertical artifacts that originate from a point along the pleura-line and lie perpendicular to the latter, show a high correlation with a wide range of pathological conditions of the lung. Their presence has been linked to an increase in extravascular lung water, interstitial lung diseases, cardiogenic and non-cardiogenic lung edema, interstitial pneumonia and lung contusion [1], [3], [4], [5]. In all these conditions, the normal structure of the lung is modified, and the size and spatial distribution of the air spaces are altered.

In working conditions where the time to evaluate the ultrasound videos in search of B-lines is scarce, a tool able to support the clinicians in this task may reveal extremely helpful. To this end, we employ modern deep learning strategies and train a fully convolutional neural network to perform this task. Weakly-supervised B-line localization is then performed by calculating neural attention maps through class-activationmapping [8]: only information on the presence or absence of B-lines in a given frame is provided for learning, after which the method automatically derives a map indicating the location of the artifact in the frame. This map reflects the importance of a given pixel with respect to the classification process of that frame as a frame with or without B-lines. The method is first tested on B-mode images of dedicated ultrasound lungmimicking phantoms able to reproduce A- and B-lines artifacts [9]. Then, results on a set of clinical B-mode images are shown, which confirm the capability of the proposed method to identify the presence of B-lines also in clinical data.

In the literature, different automated approaches have been proposed for B-line detection [10], [11], [12], [13]. Others have applied a data-driven approach and opted to, e.g., leverage a single-shot convolutional neural network to predict bounding boxes for B-lines[14]. The authors train their network on swine lung pathology models with full expert annotations of these bounding boxes. A similar approach was then used to diagnose pneumonia in patients [15]. In contrast, 
the method we present in this paper is the first to explore weakly-supervised deep learning techniques, requiring only a single label per frame for training, and that has been applied and tested both in-vitro and in-vivo [16]. Notably, the proposed method enables B-line detection in real-time, reaching an inference rate of 276 frames/second when exploiting GPU acceleration (Titan Xp, NVIDIA).

Beyond the intuitive task of detecting and localizing Blines for the purpose of clinical assessment, the presented approach can also serve as a region-of-interest selector for further quantitative analysis of segments in the ultrasound data that contain the most relevant information [9]. This does not only reduce the computational load of such operations, and thus the time needed to present a result to the clinician, it may also aid the specificity of the system by automatically discarding irrelevant signals.

The paper is organized as follows. In sections II-A to II-B2, the phantom design, the ultrasound equipment and data acquisition protocol are described. Then, in sections II-C and II-D, the adopted neural network architecture and training strategy are presented. Our approach to weakly-supervised localization from supervised detection is given in section II-E. The results are presented in section III, and finally, in section IV these results are discussed and conclusions derived.

\section{Methods}

\section{A. Lung Mimicking Phantoms}

Two different populations of mono-disperse air-filled microbubbles having diameter equal to 170 and $80 \mu \mathrm{m}$, respectively, were used to fabricate two distinct populations of lung mimicking phantoms. Both sizes were chosen to attain a cluster of air-bubbles which were smaller in size than a normal alveolar sac, whose diameter is approximately $280 \mu \mathrm{m}$ [17], thus mimicking a pathological condition where a reduction of the volume of the air spaces is induced by, e.g. the presence of liquid, blood, contusion. The micro-bubbles were generated using the MicroSphere Creator (Tide Microfluidics, Enschede, the Netherlands) and were used to form bubble clouds trapped in tissue mimicking gel. Five phantoms were fabricated with each micro-bubble population [9].

\section{B. Ultrasound data acquisition}

1) ULA-OP in-vitro data: Standard B-mode ultrasound videos of the tissue mimicking phantoms were obtained using the ULA-OP [18] research platform and the LA533 (Esaote, Florence, Italy) linear-array probe. A center frequency of $4.5 \mathrm{MHz}$ and a $0.5-\mu$ s Gaussian pulse, and a 64 elements sub-aperture (both in transmit and receive mode) were used for imaging. Similarly to a standard clinical examination, the ultrasound videos were acquired by manually operating the ultrasound probe, and the tissue mimicking phantoms were scanned as to cover the entire bubble cloud (2 to $3 \mathrm{~cm}$ in diameter). These phantoms showed capable to reproduce the typical $\mathrm{A}$ - and $\mathrm{B}$-line artifacts observable in lung ultrasound images [9]. Figure 1 shows how the phantoms were fabricated, together with examples of ultrasound images showing $\mathrm{A}$ - and B-line artifacts, respectively. Ten phantoms were scanned, generating 10 ultrasound videos for a total of 3162 frames, which were stored for further analysis. Subsequently, video-frames were labeled as either 'B-line' or 'no B-line' by a clinical lung ultrasound expert with 20 years of experience. Four of these data-sets were used as a hold-out test set for evaluation of detection performance. All acquired B-mode scans were re-scaled to $256 \times 256$ pixels and normalized between 0 and 1 prior to the analysis.

2) ULA-OP in-vivo data: The same instrumentation and imaging modality as described above was used to acquire ultrasound videos in a clinical setting. Data as acquired from 10 patients (obtained within the study number 1089 comitato etico sperimentazione clinica CEAVNO [ethical committee for clinical trial]) were used to test the performance of the algorithm described in this work. In total, 15 ultrasound videos for a total of 1552 frames were used for the analysis and labeled according to II-B. Nine scans obtained from six patients were used for training, while six scans from the four other patients were used as a separate hold-out test set. As before, all acquired B-mode scans were re-scaled to $256 \times 256$ pixels and normalized between 0 and 1 .

3) Toshiba in-vivo data: In addition, we acquired freehand ultrasound videos (from the same patient group as described above) with a commercial clinical system, i.e. the Aplio XV (Toshiba, Tokyo, Japan) scanner. The system was used together with an 11L5 linear-array probe, recording a total of 4218 frames across 12 scans. Of these, 7 were used for training, and the remaining 5 for testing. All acquired B-mode scans were re-scaled to $256 \times 352$ pixels and normalized between 0 and 1 .

\section{Neural network architecture for B-line detection}

We designed a convolutional neural network (CNN) [19] consisting of 6 convolutional blocks, each comprising two convolutional layers and a $2 \times 2$ max pool operation. Convolutional layers were followed by a ReLU activation to introduce nonlinearity while mitigating vanishing gradients [20]. The output of this sequence of nonlinear convolutional transformations was then spatially average-pooled to yield a single feature-vector representation per image. This feature vector was then processed by two fully-connected layers followed by a Softmax activation to assign scores to the two classes (B-line or no B-line present). An overview of the network architecture is given in Figure 2 and further detailed in Table I. The use of global aggregation (through pooling) after the convolutional layers promotes learning of a set of explicit feature detectors of which the activation in the resulting feature maps relates directly to the two output classes via the subsequent fully connected layers. These feature maps, and their relation with the Softmax score for the positive class (B-line present in the image) will then be used for localization, as described in Section II-E.

\section{Training strategy}

A training batch consisted of 32 randomly selected images containing B-lines, and 32 others without, which were then 
Micro-bubble microscope images
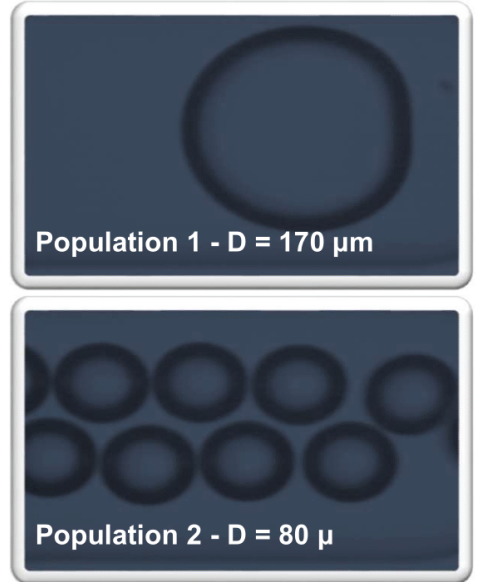

Gelatin/Bubble cloud Interface

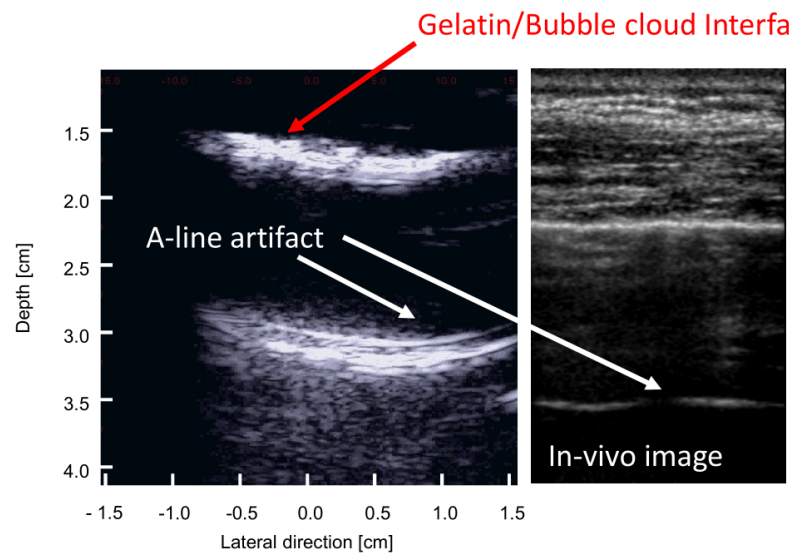

Drawing

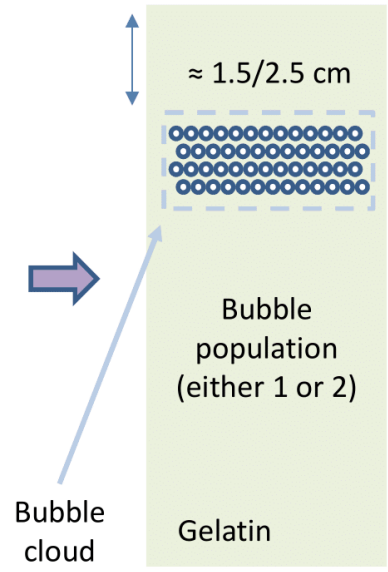

Picture

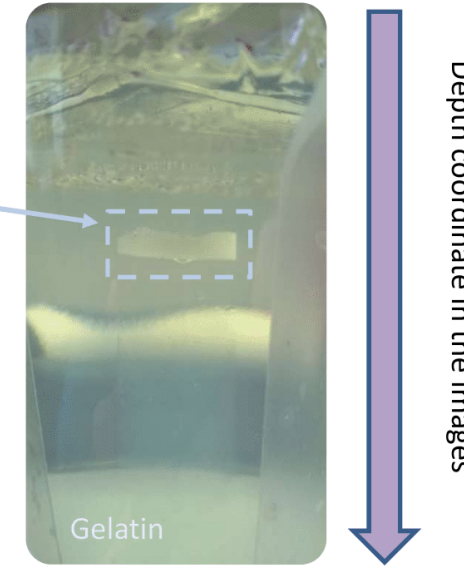

Figure 1: Micro-bubble microscope images of population 1 and 2 (top left), together with a drawing and a picture of a phantom (top right). The direction of the depth coordinate with respect to the phantom structure is also indicated. Examples of ultrasound images obtained from different phantoms and showing A- (bottom left) and B-line (bottom-right) artifacts are also shown.

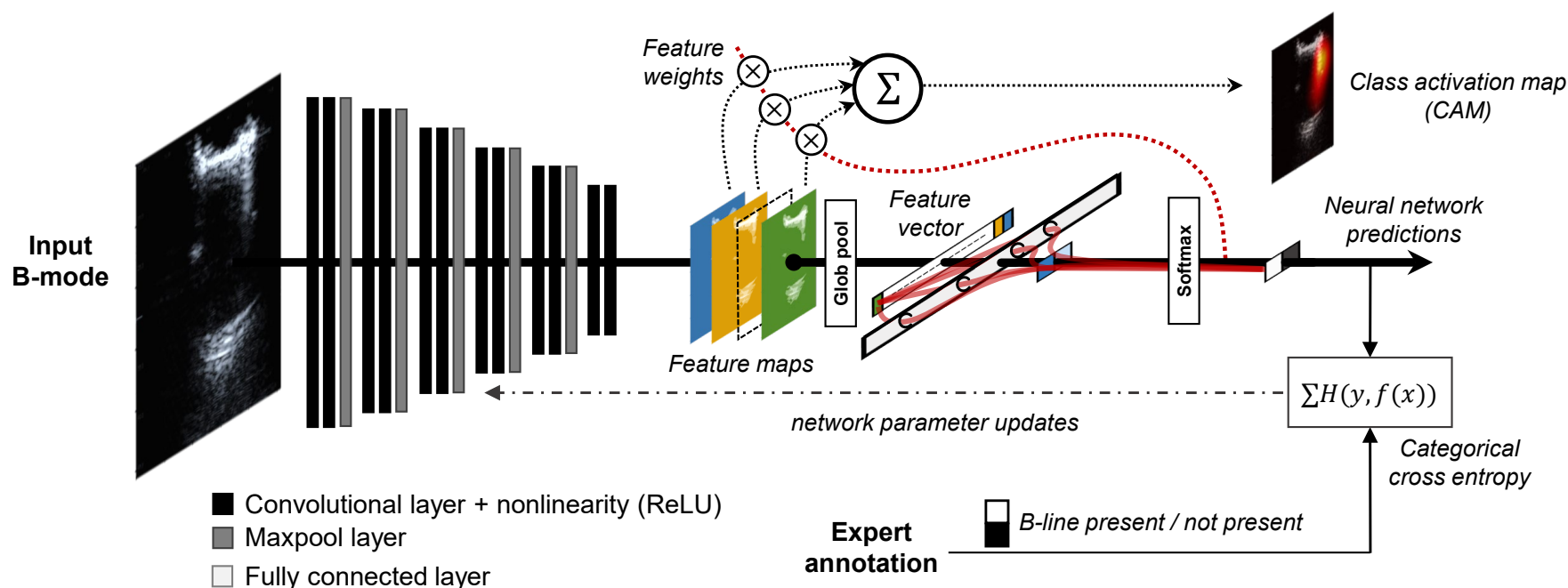

Figure 2: Overview of the proposed method for B-line detection and weakly-supervised localization through class activation mapping. The latter is a relevance-weighted combination of the convolutional feature maps, with the weights dictated by the gradients of features (global-average-pooled feature maps) with respect to the positive class. 
heavily manipulated on-line to attain high data diversity during training, and therewith generalization at inference. This manipulation included varying degrees of affine transformations, elastic warping, cropping, and blurring, and contrast distortion,

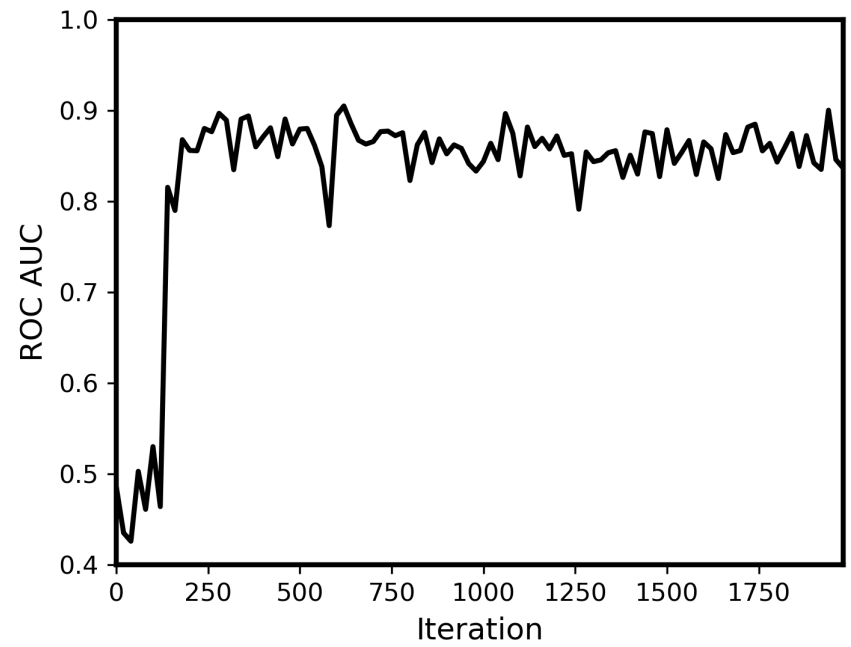

Figure 3: Receiver-operator-characteristics (ROC) curve area (AUC) on the ULA-OP in-vivo test set as a function of train iterations. An AUC equal to 1 implies an ideal performance, where both Sensitivity and Specificity are equal to 1 .

Table I: Architectural details of the adopted convolutional neural network for B-line detection.

\begin{tabular}{ccccc}
\hline \hline$\#$ & Type & Kernel dim & Activation & Output shape \\
\hline 1 & Convolutional & $3 \times 3$ & ReLU & $(256,256,32)$ \\
2 & Convolutional & $3 \times 3$ & ReLU & $(256,256,32)$ \\
3 & Max pooling & $2 \times 2$ & - & $(128,128,32)$ \\
\hline 4 & Convolutional & $3 \times 3$ & ReLU & $(128,128,64)$ \\
5 & Convolutional & $3 \times 3$ & ReLU & $(128,128,64)$ \\
6 & Max pooling & $2 \times 2$ & - & $(64,64,64)$ \\
\hline 7 & Convolutional & $3 \times 3$ & ReLU & $(64,64,64)$ \\
8 & Convolutional & $3 \times 3$ & ReLU & $(64,64,64)$ \\
9 & Max pooling & $2 \times 2$ & - & $(32,32,64)$ \\
\hline 10 & Convolutional & $3 \times 3$ & ReLU & $(32,32,64)$ \\
11 & Convolutional & $3 \times 3$ & ReLU & $(32,32,64)$ \\
12 & Max pooling & $2 \times 2$ & - & $(16,16,64)$ \\
\hline 13 & Convolutional & $3 \times 3$ & ReLU & $(16,16,128)$ \\
14 & Convolutional & $3 \times 3$ & ReLU & $(16,16,128)$ \\
15 & Max pooling & $2 \times 2$ & - & $(8,8,128)$ \\
\hline 16 & Convolutional & $3 \times 3$ & ReLU & $(8,8,128)$ \\
17 & Convolutional & $3 \times 3$ & ReLU & $(8,8,128)$ \\
18 & Average pooling & $8 \times 8$ & - & 128 \\
\hline 19 & Fully-connected & - & ReLU & 256 \\
20 & Fully-connected & - & Softmax & 2 \\
\hline \hline & & & & \\
\hline
\end{tabular}

as well as the addition of normally distributed white noise. We regularized the model by using dropout during training, with neurons dropped with a probability of 0.5 in dense layers, and a probability 0.3 in convolutional layers [21]. The model acts as an ensemble of models during testing. The neural network parameters were then learned by maximizing log-likelihood using the Adam [22] optimizer with a learning rate of $1 e^{-3}$, stochastically minimizing the cross-entropy between annotated labels and predictions.

\section{E. Weakly-supervised localization by class-activation map-} ping

Class activation mapping (CAM) enables interpretation of $\mathrm{CNN}$ predictions by revealing regions in the image that trigger a specific classification decision. Since B-lines are local image phenomena, we here exploit CAM to perform weaklysupervised B-line localization directly from the class annotations, without requiring e.g. fully annotated segmentations. To this end, we specifically leverage gradient-weighted CAM (grad-CAM) [8], a broadly applicable variant of CAM for which we first compute the gradients of the positive class score $y_{\text {pos }}$ with respect to the pixels in each image feature map, as obtained after the last convolutional layer in the CNN. Their spatial averages reflect the importance of the $k^{\text {th }}$ feature map $A_{k}$ for B-line detection, captured by the weights $w_{k}$ :

$$
w_{k}=\frac{1}{M \times N} \sum_{i=1}^{M} \sum_{j=1}^{N} \frac{\partial y_{\text {pos }}}{\partial A_{k}(i, j)},
$$

where $M$ and $N$ denote respectively the width and height of the maps. For the architecture defined in Table I, $M$ and $N$ are both 8 . The weights are then used to construct a class activation map $C$ for every input image, being an importanceweighted combination of the corresponding CNN feature maps followed by a $R e L U$ activation, further scaled by the class scores to reflect the overall B-line likelihood:

$$
C=y_{p o s} \operatorname{Re} L U\left(\sum_{k=0}^{K-1} w_{k} A_{k}\right),
$$

where $K$ denotes the amount of feature maps, here being 128 (see Table I). We expect the feature maps to reflect specific visual patterns, with each pixel activated by the presence of such a pattern (e.g. the reverberant appearance of a B-line) within its receptive field [8].

\section{RESULTS}

Model convergence for the ULA-OP data is indicated in Figure 3, displaying the Receiver-Operator-Characteristics (ROC) curve area for B-line detection on the test set as a function of the number of train iterations. Saturating at around 0.9, perfect classification is not achieved for the test set, for a large part attributed to the ambiguity of classifying and annotating frames around the onset and fading of B-lines. Similar results have been obtained for the Toshiba data.

Several illustrative B-mode images from four phantoms in the hold-out test set are given in Figure 4, clearly displaying the B-line artifacts. Qualitatively, detected B-lines are 

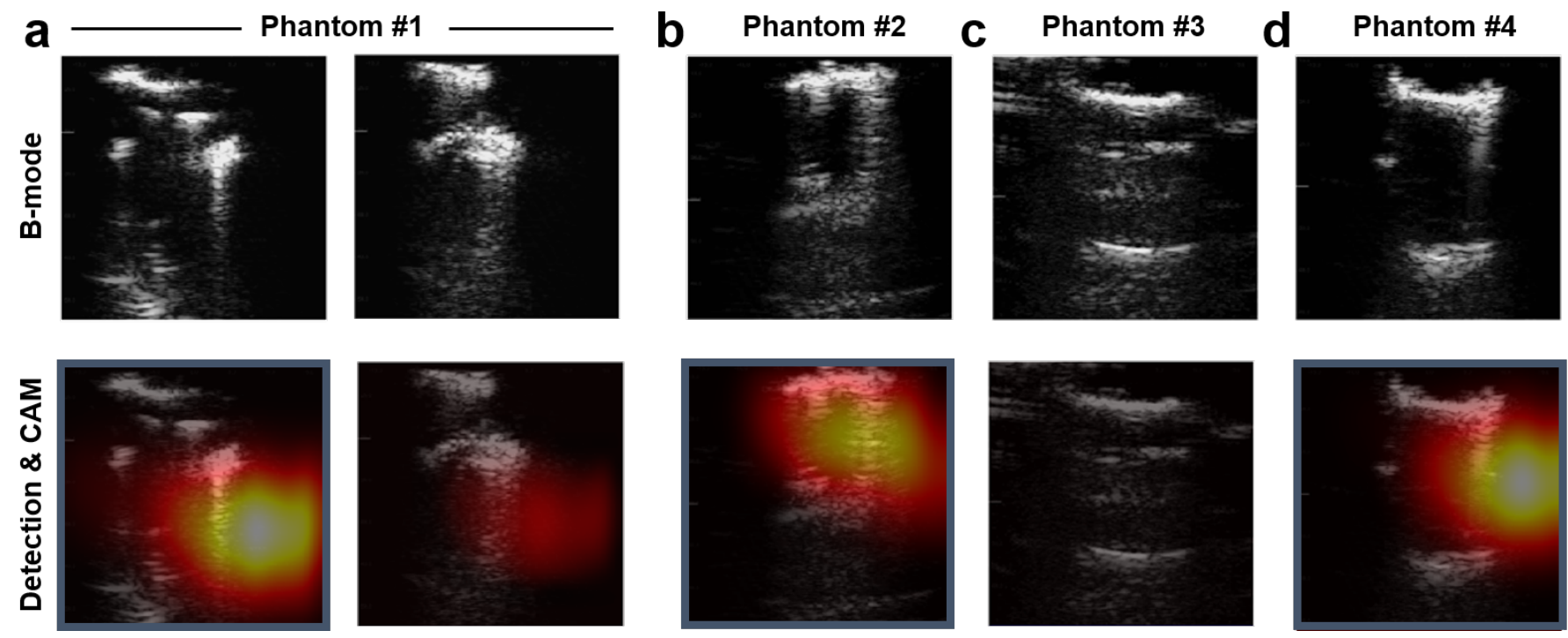

Figure 4: B-mode input data (top) and corresponding class activation maps (CAM) of B-line detection by the deep neural network for in-vitro acquisitions in 4 test phantoms (a-b). Blue boxes indicate detection.

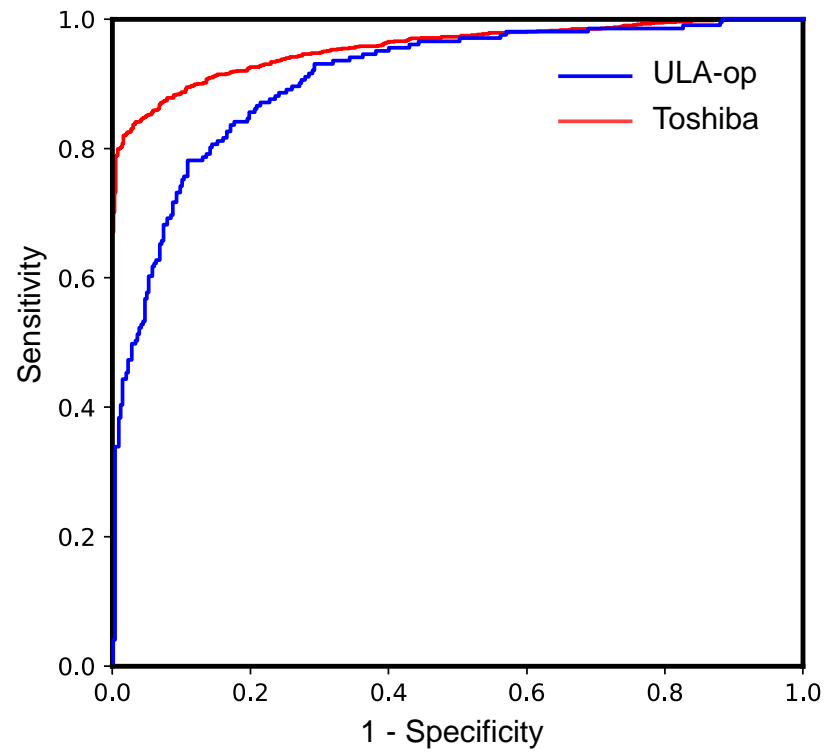

Figure 5: Receiver-operator-characteristics (ROC) curve for the ULA-OP and Toshiba in-vivo test sets, respectively.

adequately localized through CAM. When B-lines are less prominent, e.g. when moving out of plane, activation maps also dysplay a reduced intensity, following the fading of the artifact (Figure 4a).

In Figures 6 and 7, we show how this translates towards clinical application using the two different ultrasound scanners - the ULA-OP research platform and a clinical Toshiba system, respectively - displaying how the proposed method enables robust detection and simultaneous weakly-supervised localization of B-lines, not requiring expert-annotated segmentation or delineation. The performance characteristics of both scanners are visualized in Figure 5 through ROC curves.
Table II provides an overview of the overall frame-wise classification performance, reaching an in-vitro accuracy, sensitivity and specificity of $0.917,0.915$, and 0.918 , respectively. These numbers are $0.839,0.786$, and 0.868 for the ULA-OP in-vivo measurements, and 0.892, 0.871, 0.930 for the Toshiba in-vivo scans. Sensitivity and specificity are well-balanced, which can be attributed to training on batches with an equal amount of samples from both classes. The B-line detection rate as assessed by a clinical lung ultrasound specialist using the entire CAM videos was as high as $91.25 \%$. For the Toshiba invivo data, we performed an additional 3-fold cross-validation (each fold consisting of 8 train sets and 4 test sets), yielding an average accuracy, sensitivity, specificity, and area under the ROC curve of $0.832,0.856,0.697$, and 0.874 , respectively.

Higher resolution class activation maps can be obtained by using a convolutional architecture that exhibits less pooling (downsampling) prior to global average pooling of the feature maps. Figure 8 shows such maps for a network in which the last 2 max pooling layers (see Table I) have been removed, illustrating how this permits detection of multiple B-lines.

\section{CONCLUSIONS AND Discussion}

Lung ultrasound imaging is nowadays an established tool used to evaluate the condition of the lung [3]. Notwithstanding the poor understanding on the actual mechanism behind the formation of the artefactual patterns that appear in lung ultrasound

Table II: B-line detection performance in terms of accuracy (ACC), sensitivity (SEN), specificity (SPC), negative predictive value (NPV), and positive predictive value (PPV).

\begin{tabular}{cccccc}
\hline \hline Dataset & ACC & SEN & SPC & NPV & PPV \\
\hline ULA-OP in-vitro & 0.917 & 0.915 & 0.918 & 0.950 & 0.864 \\
ULA-OP in-vivo & 0.839 & 0.786 & 0.868 & 0.882 & 0.763 \\
Toshiba in-vivo & 0.892 & 0.871 & 0.930 & 0.798 & 0.958 \\
\hline \hline
\end{tabular}




\section{a}

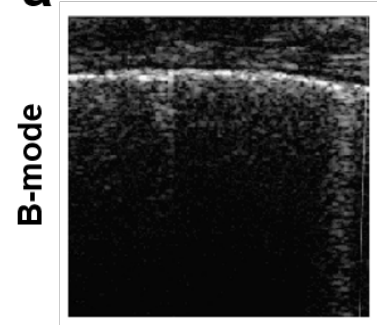

Patient \#7
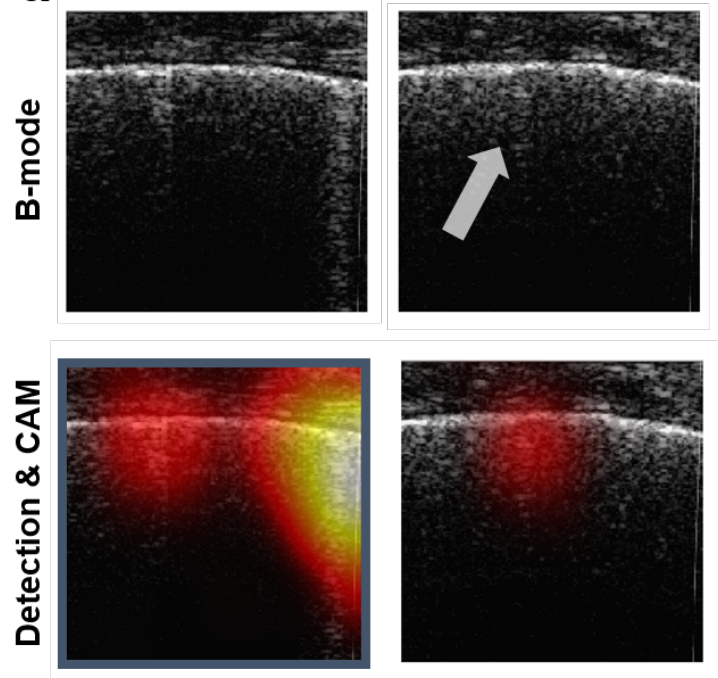

b
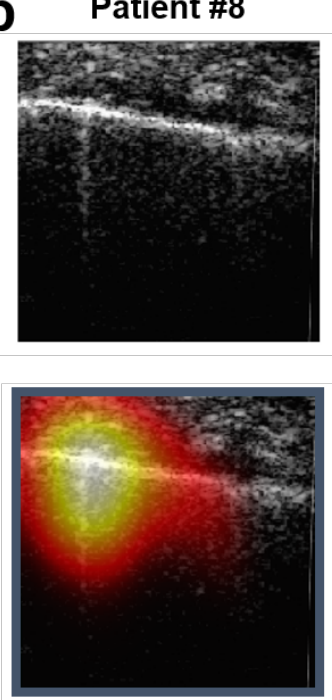
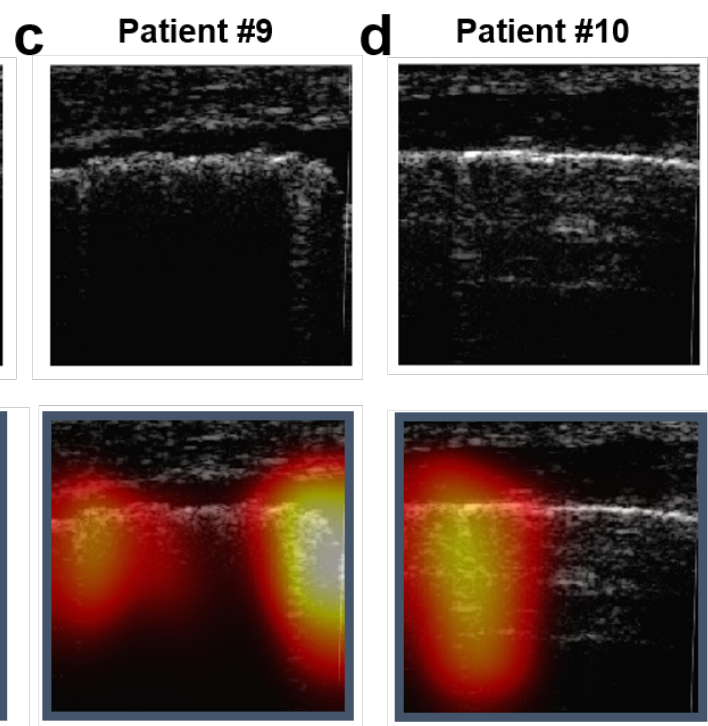

Figure 6: B-mode input data (top) and corresponding class activation maps (CAM) of B-line detection by the deep neural network for in-vivo acquisitions in 4 test patients (a-d). The activation map intensity indicates how relevant a specific pixel was for classifying the frame as one that contains a B-line, and therefore serves as a proxy for local B-line presence. Blue boxes indicate frame-based detection. The white arrow indicates a B-line in a wrongly classified image, which nevertheless appears (though less pronounced) in the CAM. See supplementary materials for the corresponding videos.

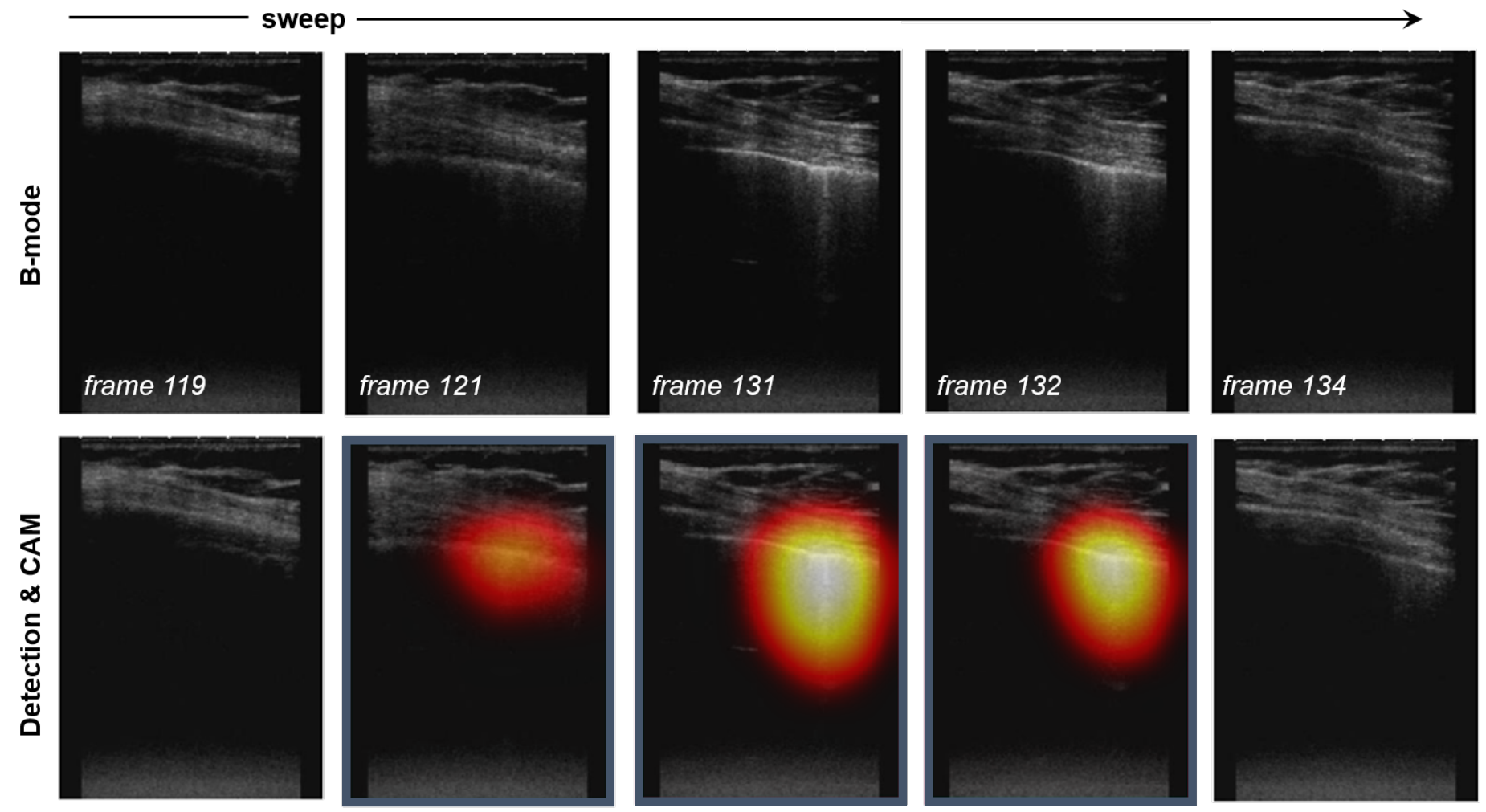

Figure 7: B-mode input data (top) and corresponding class activation maps (CAM) of B-line detection by the deep neural network for an illustrative in-vivo sweep using the clinical Toshiba ultrasound system, showing how B-lines that enter the imaging plane are well-indicated by the proposed method. Blue boxes indicate detection. See supplementary materials for the corresponding video.

images in the presence of an alteration of the lung structure, the simple identification and localization of such patterns (e.g.
B-lines) is already providing important diagnostic information for the clinician.

In this paper, we present an image analysis method based on 

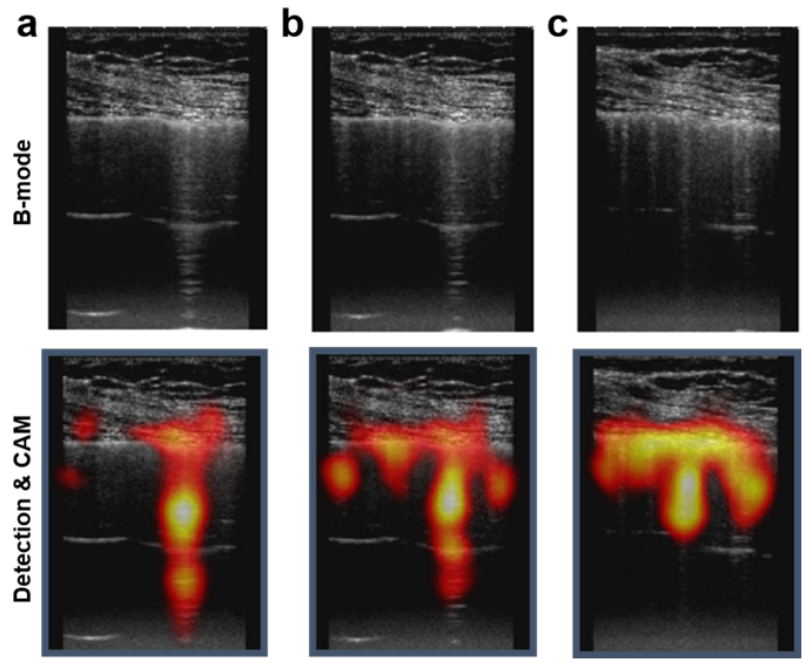

Figure 8: Clinical B-mode input data obtained using the Toshiba ultrasound system (top) and corresponding class activation maps (CAM) for frames containing multiple B-lines.

convolutional neural networks, which was developed to detect and localize B-lines in an ultrasound video. The method was tested both on in-vitro and in-vivo data, with good results in terms of accuracy, sensitivity, specificity, negative and positive predictive value. In-vivo experiments were performed using two different scanners: a research platform (ULA-op) and a clinical system (Aplio XV, Toshiba). On the former, we observed a performance drop compared to the in-vitro data, which may be due to:

1) The absence of motion in the in-vitro data. In fact, the bubble clouds may only move due to the pressure exercised by the probe while scanning. Differently, for the clinical data breathing affects significantly the presence and stability of Blines among several consecutive frames.

2) The only source of scattering for the in-vitro data are the bubble clouds, while intercostal tissue layers are also clearly present between the probe and the lung surface when scanning patients. This adds additional complexity and variability to the patterns present in the image.

Overall the inferior performance on in-vivo data may be related to the physical limitations of the phantom model. However, when assessing in-vivo performance on the clinical system, we observed retainment of the original in-vitro scores, yielding an ROC curve area of 0.957 compared to 0.909 on the research platform. We hypothesise that the higher image quality of this high-end clinical system plays an important role in achieving this performance gain.

Notably, the proposed method enables B-line detection in real-time, reaching an inference rate of 276 frames/second when exploiting GPU acceleration (Titan Xp, NVIDIA). Limitations of this study include the small number of patients enrolled from one single center (10) and the small number of videos analyzed (27).

The presented method for localization is 'weakly supervised', i.e. only image-based class labels were used to achieve localization, thereby alleviating annotators from the tedious and time-consuming task of manual delineation and segmentation of the B-lines. Relying only on image-level classes moreover mitigates the influence of inter- and intra-annotator variability in delineation, which may benefit training of the neural network and its robustness at inference. In [23], segmentation of ambiguous (but plausible) delineations from multiple readers was managed through the use of a generative segmentation model. Here we implicitly produce localizations by leveraging gradient-based class activation mapping [8]. A similar approach to weakly-supervised segmentation was presented in [24].

Spotting and identifying the part of the images where a B-line artefact is present can serve not only the clinician's workflow, but also enable dedicated region-of-interest-based processing of a subset of data to extract additional quantitative information of clinical relevance [9], [25]. In fact, the specificity of lung ultrasound B-lines is suboptimal: in addition to pulmonary congestion, these are visible in pulmonary fibrosis, flogistic or granulomatous lung interstitial diseases, atelectasis, lymphangitis, lung contusion, cardiac failure and acute respiratory distress syndrome. Dedicated signal processing approaches may instead have the power to discriminate between different pathological conditions [9]. Future work will focus on the characterization and differentiation of the detected B-line artifacts to enable adequate phenotyping of various lung pathologies [5]. Of particular interest is the development of a method able to automatically differentiate between pathological conditions where the original lung structure is altered but substantially intact (e.g. cardiogenic pulmunary edema) from conditions where the lung structure is not anymore anatomically intact (e.g. acute respiratory distress syndrome) [26].

\section{ACKNOWLEDGEMENTS}

The authors want to acknowledge the FTGM Pisa, M.D. Renato Prediletto, and M.D. Gino Soldati for access to the clinical data and for the scoring of the ultrasound videos. The authors would also like to thank the NVIDIA Corporation and its academic GPU program for donating a Titan X Pascal which greatly facilitated the research described in this work.

\section{REFERENCES}

[1] D. Lichtenstein, G. Meziere, P. Biderman, A. Gepner, and O. Barre, "The comet-tail artifact: an ultrasound sign of alveolar-interstitial syndrome," American journal of respiratory and critical care medicine, vol. 156, no. 5, pp. 1640-1646, 1997.

[2] D. Lichtenstein and G. Mzire, "Relevance of lung ultrasound in the diagnosis of acute respiratory failure: the blue protocol." Chest, vol. 144, no. 2, pp. 117-125, 2008.

[3] G. Volpicelli, M. Elbarbary, M. Blaivas, D. Lichtenstein, G. Mathis, A. Kirkpatrick, L. Melniker, L. Gargani, V. Noble, G. Via et al., "International liaison committee on lung ultrasound (ilc-lus) for international consensus conference on lung ultrasound (icc-lus). international evidence-based recommendations for point-of-care lung ultrasound," Intensive Care Med, vol. 38, no. 4, pp. 577-591, 2012.

[4] G. Soldati, M. Demi, R. Inchingolo, A. Smargiassi, and L. Demi, "On the physical basis of pulmonary sonographic interstitial syndrome," Journal of Ultrasound in Medicine, vol. 35, no. 10, pp. 2075-2086, 2016.

[5] G. Soldati, M. Demi, A. Smargiassi, R. Inchingolo, and L. Demi, "The role of ultrasound lung artefacts in the diagnosis of respiratory diseases," Expert review of respiratory medicine, no. just-accepted, 2019. 
[6] G. Soldati, V. Giunta, S. Sher, F. Melosi, and C. Dini, "Synthetic comets: a new look at lung sonography." Ultrasound in Medicine and Biology, vol. 37, no. 4, pp. 1762-1770, 2011.

[7] G. Soldati, A. Smargiassi, R. Inchingolo, S. Sheer, R. Nenna, S. Valente, C. Inchingolo, and C. GM., "Lung ultrasonography may provide an indirect estimation of lung porosity and airspace geometry." Respiration, vol. 88, no. 6, pp. 458-468, 2014.

[8] R. R. Selvaraju, M. Cogswell, A. Das, R. Vedantam, D. Parikh, and D. Batra, "Grad-cam: Visual explanations from deep networks via gradient-based localization," in Proceedings of the IEEE International Conference on Computer Vision, 2017, pp. 618-626.

[9] L. Demi, W. van Hoeve, R. J. van Sloun, G. Soldati, and M. Demi, "Determination of a potential quantitative measure of the state of the lung using lung ultrasound spectroscopy," Scientific reports, vol. 7, no. 1, p. 12746, 2017.

[10] L. J. Brattain, B. Telfer, A. Litelpo, and N. V.E., "Automated b-line scoring on thoracic sonography," Journal of Ultrasound in Medicine, vol. 32, no. 12, pp. 2185-2190, 2013.

[11] F. e. a. Corradi, "Computer-aided quantitative ultrasonography for detection of pulmonary edema in mechanically ventilated cardiac surgery patients," Chest, vol. 150, no. 3, pp. 640-651, 2016.

[12] N. Anantrasitichai, W. Hayes, M. Allinovi, D. Bull, and A. Achim, "Line detection as an inverse problem: Application to lung ultrasound imaging," IEEE transactions on Medical Imaging, vol. 36, no. 10, pp. 2045-2056, 2017.

[13] R. Moshavegh, K. Hansen, H. Moller-Soresen, M. Nielsen, and J. J. A. "Automatic detection of b-lines in in vivo lung ultrasound," IEEE transactions on Ultrasonics Ferroelectrics and Frequency Control, vol. 66, no. 2, pp. 309-317, 2019.

[14] S. Kulhare, X. Zheng, C. Mehanian, C. Gregory, M. Zhu, K. Gregory, X. Hua, M. J., and B. Wilson, "Ultrasound-based detection of lung abnormalities using single shot detection convolutional neural networks," Lecture Notes in Computer Science book series, vol. 11042, pp. 65-67, 2018.

[15] X. Zheng, S. Kulhare, and C. Mehanian, "Feature detection and pneumonia diagnosis based on clinical lung ultrasound imagery using deep learning," Abstract in Journal of the acoustical society of america, vol. 144, 2018.

[16] R. van Slound and L. Demi, "Deep learning for automated detection of b-lines in lung ultrasonography," Abstract in Journal of the acoustical society of america, vol. 144, 2018.

[17] K. B. Sagar, T. L. Rhyne, G. S. Myers, and R. S. Lees, "Characterization of normal and abnormal pulmonary surface by reflected ultrasound," Chest, vol. 74, no. 1, pp. 29-33, 1978.

[18] E. Boni, L. Bassi, A. Dallai, F. Guidi, A. Ramalli, S. Ricci, J. Housden, and P. Tortoli, "A reconfigurable and programmable fpga-based system for nonstandard ultrasound methods," IEEE transactions on ultrasonics, ferroelectrics, and frequency control, vol. 59, no. 7, pp. 1378-1385, 2012.

[19] A. Krizhevsky, I. Sutskever, and G. E. Hinton, "Imagenet classification with deep convolutional neural networks," in Advances in neural information processing systems, 2012, pp. 1097-1105.

[20] Y. LeCun, Y. Bengio, and G. Hinton, “Deep learning," nature, vol. 521, no. 7553, p. 436, 2015.

[21] N. Srivastava, G. Hinton, A. Krizhevsky, I. Sutskever, and R. Salakhutdinov, "Dropout: a simple way to prevent neural networks from overfitting," The Journal of Machine Learning Research, vol. 15, no. 1, pp. 1929-1958, 2014

[22] D. P. Kingma and J. Ba, "Adam: A method for stochastic optimization," arXiv preprint arXiv:1412.6980, 2014.

[23] S. Kohl, B. Romera-Paredes, C. Meyer, J. De Fauw, J. R. Ledsam, K. Maier-Hein, S. A. Eslami, D. J. Rezende, and O. Ronneberger, "A probabilistic u-net for segmentation of ambiguous images," in Advances in Neural Information Processing Systems, 2018, pp. 6965-6975.

[24] Y. Wang, F. Zhu, C. J. Boushey, and E. J. Delp, "Weakly supervised food image segmentation using class activation maps," in 2017 IEEE International Conference on Image Processing (ICIP). IEEE, 2017, pp. 1277-1281.

[25] K. Mohanty, J. Blackwell, T. Egan, and M. Muller, "Characterization of the lung parenchyma using ultrasound multiple scattering," Ultrasound in medicine \& biology, vol. 43, no. 5, pp. 993-1003, 2017.

[26] G. Soldati, M. Demi, and L. Demi, "Ultrasound patterns of pulmonary edema," Annals of Translational Medicine, 2019. 